\title{
Circuit
}

Musiques contemporaines

\section{Composition orientée objet Object-oriented composition}

\section{Nicolas Bernier}

Volume 23, numéro 1, 2013

La musique des objets

URI : https://id.erudit.org/iderudit/1017210ar

DOI : https://doi.org/10.7202/1017210ar

Aller au sommaire du numéro

Éditeur(s)

Les Presses de l’Université de Montréal

ISSN

1183-1693 (imprimé)

1488-9692 (numérique)

Découvrir la revue

Citer cet article

Bernier, N. (2013). Composition orientée objet. Circuit, 23(1), 33-44.

https://doi.org/10.7202/1017210ar

\section{Résumé de l'article}

C'est par la lorgnette de sa pratique que l'artiste esquisse une démarche de création sonore ancrée dans le rapport au matériel : du rapport à l'objet sonore, à l'objet matériel, au corps sonore, au corps humain jusqu'à la création d'une nouvelle lutherie. En puisant dans une myriade de références hétérogènes, des liens sont tissés entre musique fixée et musique électroacoustique exécutée en direct et permettent d'envisager un dialogue possible entre des démarches compositionnelles souvent pensées comme radicalement opposées.
Ce document est protégé par la loi sur le droit d'auteur. L'utilisation des services d’Érudit (y compris la reproduction) est assujettie à sa politique d'utilisation que vous pouvez consulter en ligne.

https://apropos.erudit.org/fr/usagers/politique-dutilisation/ 


\section{Composition orientée objet}

Nicolas Bernier

Un jour j'irai vers l'irréel / Tester le matériel

- Alain Bashung, «L'irréel».

\section{De l'abstrait au concret}

Tout a commencé par un désir d'enregistrer le monde sonore pour le réécouter sans diversion visuelle, pour m'en approcher intimement afin de mieux le comprendre, le décortiquant dans ses plus fines particules. Il me vint rapidement le réflexe d'archiver ce monde enregistré, de le ranger comme dans de petites boîtes étiquetées afin de pouvoir revenir sur cette mémoire et, surtout, l'utiliser comme ingrédient compositionnel. La boucle se boucla quand naquit de la composition à base de sons enregistrés le besoin de conserver non pas seulement les sons, mais également leurs sources. Lorsque cela est possible, bien évidemment. Car ces sources sonores sont pour moi davantage que de simples objets anonymes producteurs de son. Ce n'est pas seulement le son de ces objets qui s'immisce dans l'antre de mon microphone, mais les objets eux-mêmes: mon entendre immatériel se nourrit de mon voir matériel.

Ma démarche s'est donc tournée vers la recherche d'un équilibre entre matière abstraite et concrète - concrète au sens où cette matière maintient un lien avec la source sonore d'où elle provient. À la recherche de la musique concrète, j'espérais naïvement trouver une musique «concrète », c'est-à-dire qui me lierait au monde matériel, aux objets physiques qui m’entourent ${ }^{1}$. De la tradition concrète, j'en suis arrivé à interpréter sur scène une musique électroacoustique reposant sur la manipulation d'objets, la présence du corps et l'invention d'instruments sans pour autant négliger l'utilisation du son fixé sur support que nous a apporté la musique concrète 2 . Ce rapport à l'objet matériel est pour moi une façon de rester en contact avec le monde tangible malgré un mode de composition principalement virtuel puisque mené à bien par l'entremise de l'ordinateur.

1. Musique concrète: nom donné par Pierre Schaeffer à sa découverte en 1948 et qui proposait "une inversion dans le sens du travail musical". II précisait: "Au lieu de noter des idées musicales par les symboles du solfège, et de confier leur réalisation concrète à des instruments connus, il s'agissait de recueillir le concret sonore, d'où qu'il vienne, et d'en abstraire les valeurs musicales qu'il contenait en puissance" (Schaeffer, 1966, p. 23).

2. Musique électroacoustique: appellation utilisée historiquement pour nommer les musiques composées avec sons de synthèse et/ou enregistrés et/ou avec traitement de signal, qu'elles soient fixées sur support ou interprétées en direct. Bien que cette dénomination ait le défaut d'être généraliste, elle possède une qualité d'ouverture sur le champ des possibles musicaux. 
3. Schaeffer décrivait ainsi l'objet sonore: "Si l'on nous présente une bande sur laquelle est gravé un son dont nous sommes incapables d'identifier l'origine, qu'est-ce que nous entendons? Précisément ce que nous appelons un objet sonore, indépendamment de toute référence causale désignée, elle, par les termes de corps sonore, source sonore ou instrument" (Schaeffer, 1966, p. 95).

4. Ibid.

5. Chion, 2009, p. 14.

6. Chion, 1993b, p. 51

7. Voir Schaeffer, 1966, p. 62.

8. Voir Flusser, 1999, p. 59.
Témoin d'une approche personnelle, ce texte explore ces allers-retours, tissant des liens entre objet sonore, objet matériel, corps sonore, corps humain et nouvelle lutherie.

\section{Du rapport à l'objet sonore}

Mon attachement à la tradition concrète est déterminant, la découverte, par pur hasard, du Traité des objets musicaux de Schaeffer (1966) ayant précédé ma connaissance de l'existence même de la musique électroacoustique. Cependant, j'ai toujours été tracassé par le fait que Schaeffer ait utilisé un vocable à résonance matérielle - musique concrète, objet sonore ${ }^{3}$ - pour communiquer le fruit de ses recherches qui traitaient plutôt de dématérialisation des sources sonores, puisque le son perçu n'avait plus à être associé à son corollaire matériel. Il s'agissait plutôt d'effectuer, comme l'écrit Schaeffer, une «écoute aveugle des effets du contenu sonore ${ }^{4}$ ». Ce vocable a nourri des générations de création sonore en induisant, au fil du temps, une confusion dans la pratique, «un malentendu qui persiste aujourd'hui encore ", déclare Michel Chion. La problématique réside ici dans le fait qu'une part de praticiens a eu naturellement tendance à associer la musique concrète ainsi que l'objet sonore avec l'enregistrement de sons acoustiques et d'objets matériels, ce qui n'est pas nécessairement le cas:

It is as a result of a disastrous shift in the meaning, for which the concrete composers are themselves partly responsible, that the term « concrete music » subsequently came to have the very restricted sense of music made with so-called concrete sounds, that is to say, with sounds originating from an acoustic source ${ }^{6}$.

Les dénominations «musique concrète » et «objet sonore » ne pouvaient que trop facilement renvoyer au monde physique et à sa mise en mémoire par la prise de son. Ces principes schaefferiens auraient-ils pu évoluer au fil des années pour s'ouvrir aux pratiques en développement? Ils ont plutôt été confinés dans le champ de la musique fixée sur support pour désigner implicitement, la plupart du temps, un type d'écriture où la matière perçue est dénuée de référent. Avec l'héritage schaefferien, nous nous devions de comprendre qu'un objet n'est pas un objet, mais un objet de perception? ${ }^{7}$. Pour paraphraser le philosophe Vilém Flusser, la théorie schaefferienne utilisait l'objet perçu pour mieux occulter l'objet matériel's.

Les artistes concevant des musiques avec des sons captés en direct n'ont pu légitimement revendiquer d'être des musiciens concrets puisque leurs sources se trouvaient sur scène, à la vue des spectateurs. Comme l'écrit Chion : «It is obvious that in order to assert itself, concrete music today is having to refuse to 
join with "real-time" .» En tant que jeune praticien, il m'aura fallu quelques années avant de comprendre que je ne pouvais m'associer à la musique concrète lorsque je me présentais sur scène avec des objets. Pourtant, «nul compositeur un peu conséquent, lorsqu'il s'engage dans la musique concrète, ne peut se soustraire à cette délicate question du figuratif sonore ${ }^{10}$ », note Chion. La même musique ne serait donc pas identique selon son contexte de présentation. Peut-être, certes, car «on ne "voit" pas la même chose quand on entend; on n' "entend" pas la même chose quand on voit ${ }^{11}$ ». Mais comme praticien qui effectue des allers-retours entre composition fixée sur support et musique électroacoustique en direct, mon travail sur scène s'effectue pourtant en parfaite continuité avec le travail de studio. Schaeffer avait pris soin de préciser son propos en avançant la notion de « corps sonore » que nous devons distinguer de l' «objet sonore». Là où l'objet sonore est un effet perceptif, donc immatériel, le corps sonore est quant à lui ce que le commun des mortels nommerait un «objet sonore», soit un objet matériel qui produit un son.

Bien que Schaeffer réfutera en 1958 le terme «musique concrète » au profit de «musique expérimentale», la terminologie originale est restée bien vivante dans le vocabulaire des artistes du sonore ${ }^{12}$. Chez le praticien qui utilise cette terminologie théorique dans la pratique, une inévitable distorsion sémantique s'est présentée au fur et à mesure que les musiciens de tradition concrète investissaient la scène de leurs objets et de leur traitement en temps réel. Créant en direct des objets sonores par la manipulation et l'enregistrement de corps sonores, comment auraient-ils pu éviter de sentir une forte affinité à la musique concrète? Pour ma part, je ne peux m'empêcher de sentir que ma pratique est concrète, puisque je compose, souvent, à partir d'objets desquels j'extrais des sons, et ce, autant en studio que sur scène, que la source soit reconnaissable ou non. Comme compositeur, je me suis toujours senti plus proche des premières études de Schaeffer où le traitement est rudimentaire que des musiques électroacoustiques développées dans les décennies suivantes avec des traitements sophistiqués. Ceux-ci m’apparaissent plutôt comme un filtre dissociant le son de sa source matérielle et de la prise de son qui demeurent des prémisses fondamentales de cette forme d'art. Ce qui m’a interpellé dans la musique électroacoustique est bel et bien ce rapport à la réalité, au matériel, à la vie, et celui qu'entretient l'humain avec les objets et la matière qui l'entourent.
9. Chion, 1993b, p. 52.

10. Chion, 2009, p. 7.

11. Chion, 1990, p. 3.

12. Voir Schaeffer, 1966, p. 62. Le terme "musique concrète" sera réintroduit en 1991 par Michel Chion dans son ouvrage L'art des sons fixés, revu en 2009 sous le titre La musique concrète, art des sons fixés. 
13. "Early musical instruments were made from "found objects" such a shells and plant parts" (Rault, 2000, p. 9).

14. Voir Christoph Cox in Young, 2002, p. 39 .

15. Pierre Henry in Chion, 1977, p. 104.

16. Dissemblablement à la situation classique du concert acousmatique où la composition préécrite est diffusée sur haut-parleurs.

17. Baudrillard, 1968, p. 105

\section{Du rapport à l'objet matériel}

L'art sonore entretient depuis des lustres cette relation à l'objet matériel: des musiques primitives ${ }^{13}$ aux futuristes italiens (Luigi Russolo, 1913), jusqu'à la Waterwalk de John Cage (1960), les instruments inventés de Harry Partch, les performances de The Sonic Arts Union dans les années 1960 ${ }^{14}$ ou encore les percussions scrap metal du groupe industriel allemand Einstürzende Neubauten. Pierre Henry, principal collaborateur de Pierre Schaeffer dans les années 1950, exprimait son intérêt pour la «musique d'objets", évoquant le passage possible de l'approche concrète du studio vers la scène:

Mais il y a un domaine où on n'a rien fait: celui que j'appelle la «musique d'objets », et c'est ça qui me tente à l'avenir. Il s'agirait de faire travailler des gens sur des corps sonores plus ou moins amplifiés, plus ou moins électrifiés et transformés - peut-être pas du tout -, et d'inventer ainsi une nouvelle lutherie - pas dans le style «Dada» des Futuristes et de leurs bruiteurs mais avec des corps sonores qu'on puisse manier, moduler, jouer, avec toute la subtilité venant d'une éducation musicale très sérieuse et du geste instrumental ${ }^{15}$.

Dans la manipulation de l'objet se trouve un moyen d'engager la musique électroacoustique dans le monde matériel, unissant le faire, le voir et l'entendre ${ }^{16}$. Mais pourquoi un artiste du son s'entêterait à entretenir une relation avec l'objet matériel alors qu'il peut aisément s'en départir dans la composition de tradition concrète? À mes yeux, cet objet est plus qu'une vulgaire chose de laquelle j'extirpe un son: il occupe mon espace de travail, il habite ma pensée au quotidien, il me raconte une histoire - son histoire réelle que je connais ou encore son histoire fictive que je me plais à inventer - à laquelle se greffera la mienne. Cette attirance pour l'objet matériel me suit depuis la tendre enfance où s'est amorcé un intérêt pour la collection. C'est d'ailleurs «chez l'enfant le mode le plus rudimentaire de maitrise du monde extérieur: rangement, classement, manipulation ${ }^{17}$ ", écrit Jean Baudrillard. Comme si je trouvais dans ce rapport à l'objet - et à la possibilité de l'organiser dans le monde extérieur - un contrepoids au fouillis intérieur, aux idées compositionnelles qui foisonnent dans un perpétuel maelström sur lequel je n’ai que peu de contrôle.

Si je collectionne les sons pour composer, je collectionne d'abord et avant tout des objets, je collectionne le monde que j'habite et qui m'habite. Composer, collectionner et organiser sont, au sein de ma pratique, des démarches intrinsèquement liées. Il s'agit de chercher, de trouver péniblement la bonne pièce (tout autant que le bon son), de rêver de l'objet manquant, de faire le tour des brocantes et de toucher la matière avec mes mains pour ensuite former des structures, construire des systèmes organisa- 


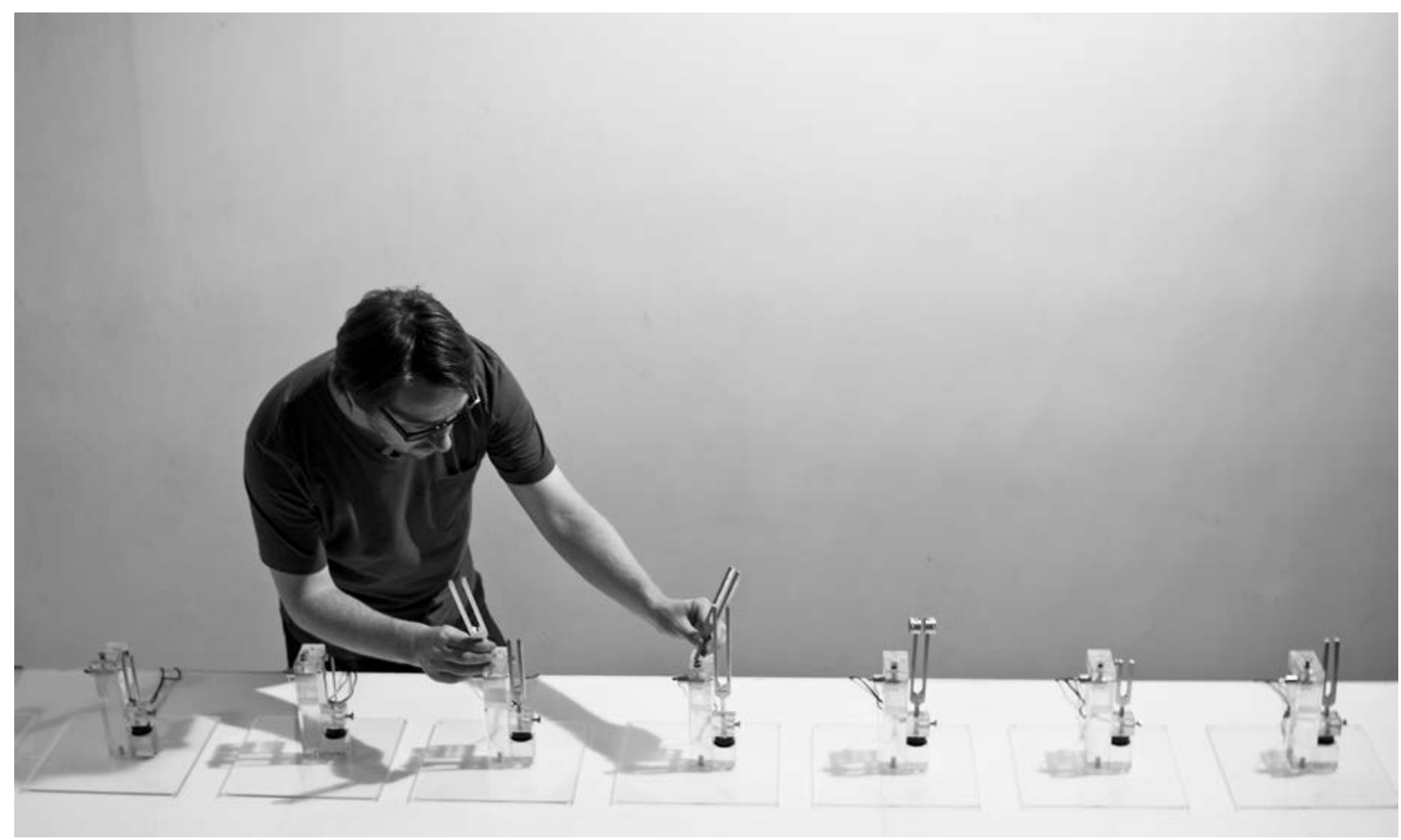

tionnels, et ce, autant dans le monde physique que dans le monde sonore. Par exemple, ce sont des diapasons qui sont collectionnés durant le processus créatif menant à la performance sonore frequencies (a) (2012) - des diapasons de toutes tailles, fréquences, origines et époques ${ }^{18}$. Je les considère étroitement liés à ma pratique, créant un pont entre son acoustique et électronique : le son du diapason - utilisé comme symbole d'une musique acoustique - se rapproche étrangement du son sinusoïdal de synthèse - comme symbole de la musique électronique. Dans cette performance, des séquences mécaniques sont lancées depuis l'ordinateur, activant des solénoïdes qui font résonner les diapasons acoustiques ${ }^{19}$. Il s'agit d'une construction formelle où le son quasi pur des diapasons est mis en relation avec des fréquences pures de synthèse.

La relation à l'objet matériel est également au centre de la majeure partie de mes musiques concrètes que j’ai regroupées sous le titre Travaux méca18. frequencies (a) a été créée le 10 octobre 2012 dans le cadre du festival Champs électriques à Ottawa.

19. Les solénoïdes sont de petits bras mécaniques activés par courant électrique. niques (2012). Dans l'œuvre Writing Machine, ce sont principalement les sons d'une machine à écrire qui sont mis en musique. Dans Liaisons mécaniques, 
20. Allard, 2012, p. 5.

21. "Anecdotique" est un qualificatif donné aux musiques électroacoustiques où les sources sont reconnaissables.

22. Allard, 2012, p. 5. L'animisme, qui désigne la tendance à attribuer un caractère vivant à des objets inanimés, représente très justement cette relation à sens unique (ibid.).

23. Baudrillard, 1968, p. 20.

24. Julian Schnabel in Le Feuvre, 2010, p. 131. il s'agit d'enregistrements de petits engrenages mécaniques à partir desquels des liens musicaux sont ficelés. Ces petits objets ont été collectionnés sur des années avant de pouvoir penser à la composition. Ces compositions lient l'objet matériel et l'objet sonore schaefferien, l'objet référentiel et sa poétisation, tout autant que sa dénaturation par le traitement de signal. On y retrouve ainsi l'objet sous sa double nature: «à la fois anthropologique lorsque défini comme artéfact et poétique lorsque proposé en tant que métaphore, emblème ou allégorie ${ }^{20}$ », tel que le note Dominique Allard. Mon intention n’est pourtant pas de composer de façon anecdotique, mais de trouver un équilibre entre la matière sonore enregistrée et sa transformation ${ }^{21}$. Là où j'espère toujours réussir à faire ressentir l'objet d'origine, il demeure primordial d'en faire ressortir des qualités sonores insoupçonnées, évitant de le présenter comme une pure documentation.

Ces préoccupations sont présentes jusque dans la présentation en concert des Travaux mécaniques. La musique concrète est traditionnellement présentée dans l'obscurité, misant sur l'écoute et non la mise en scène. Pour ma part, même lorsqu'il s'agit de musique concrète, j'aime construire une présentation visuelle qui évoque les objets utilisés dans les compositions. J'y transporte mon atelier directement sur scène: table de travail, lampes, instruments et bibelots, donnant ainsi accès au visuel et aux objets qui ont imprégné la composition de leurs esprits. Car si mes compositions prennent vie par ces objets, je vis avec ces objets et ils vivent avec moi, me rapprochant peut-être d'un animisme où «l'humain [...], par la projection de ses fantasmes, de ses désirs, humanise et attribue une âme à l'objet ${ }^{22}{ }$. Ces mêmes œuvres présentées sur scène, mais sans objets, ne transmettraient pas le même propos, leur simple présence participant à communiquer l'œuvre. Les objets magnifient ici l'important fait matériel au sein des compositions et forment un liant entre le corps du musicien, les idées qui habitent l'œuvre et le monde matériel. Comme l'indique Baudrillard: «Êtres et objets sont d'ailleurs liés, les objets prenant dans cette collusion une densité, une valeur affective qu'on est convenu d'appeler leur "présence"23.»

Ainsi, j’intègre des éléments de ma propre vie et, ipso facto, des éléments des compositions au sein de leurs présentations. Dans ce statement, l'artiste états-unien Julian Schnabel explique le phénomène de façon poétique:

I want my life to be embedded in my work, crushed into my painting like a pressed car. If it's not, my work is just some stuff. When I'm away from it, I'm crippled. Without my relationship to what may seem like these inanimate objects, I am just an indulgent misfit. If the spirit of being isn't present in the face of this work, it should be destroyed because it's meaningless ${ }^{24}$. 


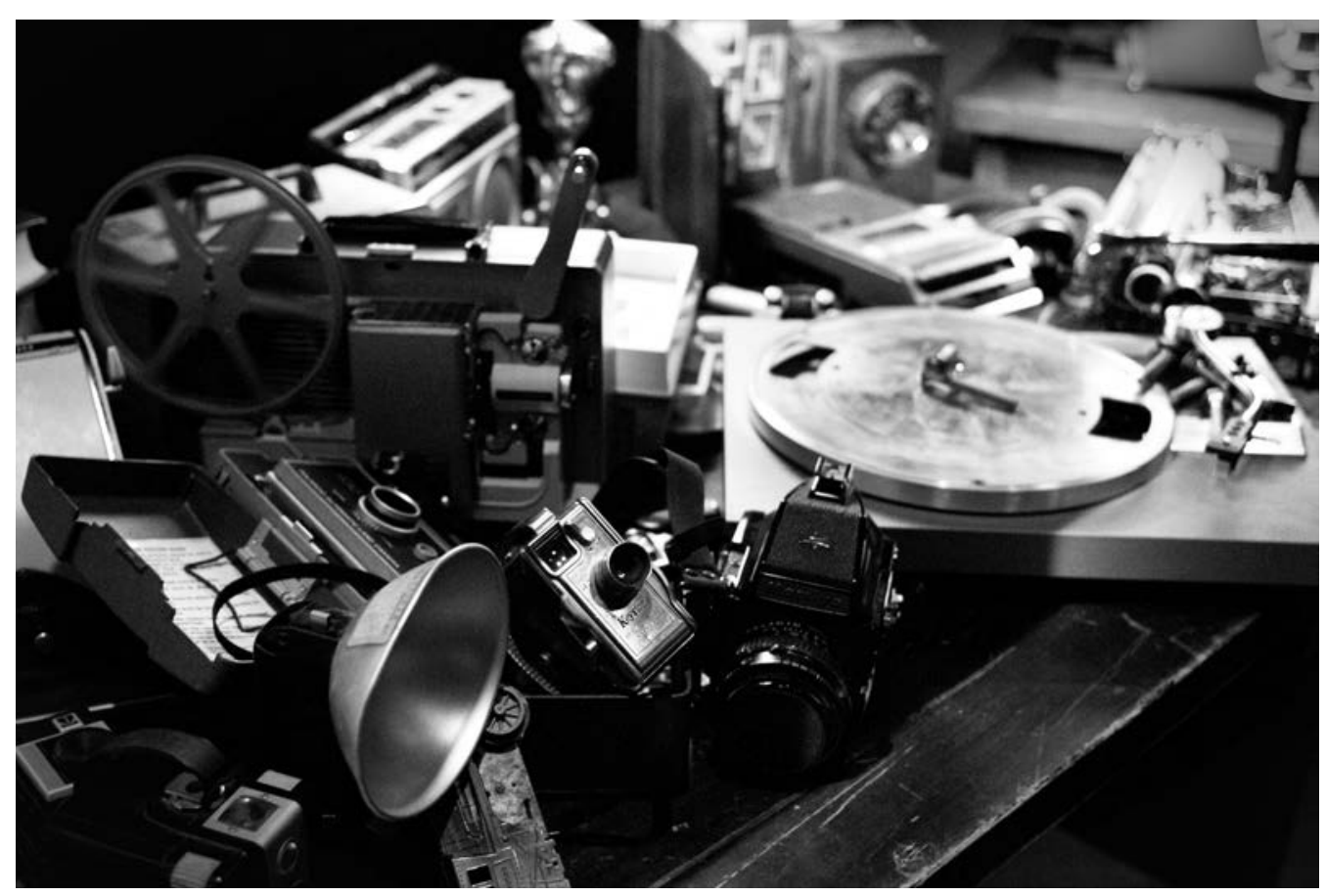

L'importance donnée au matériel provient chez moi d'une réaction à la dématérialisation des éléments compositionnels (l'objet sonore schaefferien), de la composition en soi (composition par ordinateur) et de la performance dans les musiques électroacoustiques (composition diffusée sur support, sans interprétation). Mais cette relation à l'objet matériel, au corps sonore, ne se fait qu'avec la présence du corps humain.

\section{Du rapport au corps}

The measure of a work of art is whether one can sense in it the presence of the artist's body. If so, then it is a success, and if not, it's a failure. - Pierre Hébert.

Je suis arrivé dans le monde électroacoustique à l'orée du numérique où tout semblait loin de la matière physique: génération de sons de synthèse, composition par ordinateur, envoi des pièces par internet, jusqu'à la diffusion de l'œuvre qui ne nécessite pas la présence du compositeur puisqu'elle peut être jouée par l'intermédiaire d'un support standard (CD, DVD, DAT, etc.). Lorsque présenté sur scène, l'instrument de musique par excellence était désormais le laptop, tel que l'émet Chris Salter dans son livre Entangled: 
25. Salter, 2010, p. 214.

26. Ibid., p. xxi.

27. Baudrillard, 1968, p. 34

28. Foucault, 1966, en ligne.

29. Chion, 1993a, p. 163
The aesthetic shifts from what became known as laptop music were far greater than just neoteri technical possibilities. The shift to the laptop as the instrument of electronic sound culture signaled an even more extreme move towards an aesthetics of the digital conditioned by software and hardware ${ }^{25}$.

Dans l'introduction de son ouvrage, Salter aborde d'ailleurs cette traversée de l'ère numérique où, après des années de travail plutôt «virtuel », les artistes sont revenus à un travail où le corps humain et la matière physique redevenaient prédominants :

As euphoria for the simulated and the virtual that marked the end of the twentieth century subsides, suddenly everyone from new media artists to architects, physicists, ethnographers, archaeologiststs, and interaction designers are speaking of embodiment, situatedness, presence, and materiality. In short, everything has become performative ${ }^{26}$.

Où il y a objet matériel, il y a corps humain. Baudrillard émet même l'idée que «l'homme est lié alors aux objets ambiants par la même intimité viscérale (toutes proportions gardées) qu'aux organes de son propre corps ${ }^{27}$ ». Nul ne peut échapper à la présence du corps, pas même la musique fixée sur support, car du corps nous ne pouvons nous affranchir. Michel Foucault observe ainsi :

Je peux bien aller au bout du monde, je peux bien me tapir, le matin, sous mes couvertures, me faire aussi petit que je pourrais, je peux bien me laisser fondre au soleil sur la plage, il sera toujours là où je suis. Il est ici irréparablement, jamais ailleurs. Mon corps, c'est le contraire d'une utopie $[\ldots]^{28}$.

La relation à l'objet est pour moi intrinsèquement liée à celle du corps humain. À travers l'objet et sa manipulation, c'est ultimement le corps humain que j'espère faire ressentir dans mes compositions. Même chez celui qui compose des œuvres faites pour être entendues dans un contexte de musique de sons fixés, nous constaterons souvent cette attraction pour un travail où le matériel est manipulé. C'est le cas de Michel Chion qui, au lieu de travailler sur ordinateur, dit préférer opérer :

sur un support matériel concret que je peux manipuler, toucher, dédoubler, voire agresser et qui me stimule en me permettant un branchement physique direct sur le travail sonore, plutôt que d'utiliser l'intermédiaire d'une simulation optique ${ }^{29}$.

L’objet sera manipulé plus ou moins adroitement par un humain, car un objet manipulé par un autre objet - donc une machine - n’aura pas cette capacité de transmettre la présence du corps humain qui se manifeste, contrairement à la machine, dans son imperfection.

Arrivé dans la création audionumérique, la performance - et, par incidence, la présence humaine - s'est rapidement imposée comme une approche inévi- 
table, tranchant avec l'approche du compositeur travaillant en solo avec son ordinateur. Pour moi, enfant-du-tout-numérique, cette présence humaine se présentait même comme une curiosité, une chose rare: «Recording, we all know, has changed the way people listen to music, perform music and compose music. It has also, ironically, made the act of creating music, live and in person, a signally "special" event ${ }^{30}$ », remarque Nicolas Collins. Il me fallait donc subvertir cette aliénation du corps que semblait vouloir imposer la vie au numérique.

\section{Du rapport à l'instrument}

Une fois le corps humain engagé dans la manipulation de l'objet, une virtuosité instrumentale se développe. Ce retour à la virtuosité dans les musiques électroacoustiques était déjà souhaité par Bob Ostertag en 2002 :

Virtuosity has been out of fashion for years now, ever since the advent of punk rock, conceptual art and other movements that emphasize the idea rather than its execution. Nevertheless, virtuosity of some sort is a necessary element of almost any performance ${ }^{31}$.

Cette notion de virtuosité est plus ouverte que traditionnellement, quand l'interprète se dédiait à la pratique d'un instrument unique. L'instrument est aujourd'hui plus éclaté, se transformant au gré des projets, des trouvailles et des poussées technologiques. Bien que la recherche soit davantage axée sur le développement de nouveaux instruments et haptiques ${ }^{32}$, nombre de propositions d'artistes reposent sur les «vieilles» technologies: pensons à Ben Frost et Christian Fennesz qui créent de grandes fresques tramées en combinant ordinateur, avec sons fixés, avec leurs guitares électriques, garnies de leurs indispensables effets. Le duo Matmos conjugue les techniques de la musique concrète aux synthétiseurs. Lionel Marchetti a façonné son instrument en combinant mélangeur, microphones, flash photographique et objets. C'est, pour ma part, en m'inspirant des intonarumoris futuristes avec les projets Boîte (2008) et La chambre des machines (collaboration avec Martin Messier, 2010) que j'entreprends un travail sur une certaine virtuosité instrumentale ${ }^{33}$. Dans cette performance, les interprètes jouent sur des instruments faits de bois et de métal, confectionnés par Alexandre Landry. Le système est d'une relative simplicité: des microphones piézoélectriques dont le signal est analysé en temps réel déclenchent des traitements audio ainsi que des séquences audiovisuelles préenregistrées. La composition se déroulant en continu - sans interaction entre le programme et les interprètes -, les événements, traitements, effets et amplifications se trouvent à chaque performance au même
30. Collins, 2008, p. 7 .

31. Ostertag, 2002, p. 11

32. Pensons au Radio-Baton de Max Mathews, à The Hands de Michel Waisvisz ou encore au T-Stick de Joseph Malloch.

33. Boîte a été créée le 19 septembre 2008 dans le cadre du Mois Multi à Québec. La première de La chambre des machines a eu lieu le 6 février 2010 au festival Transmediale de Berlin. 


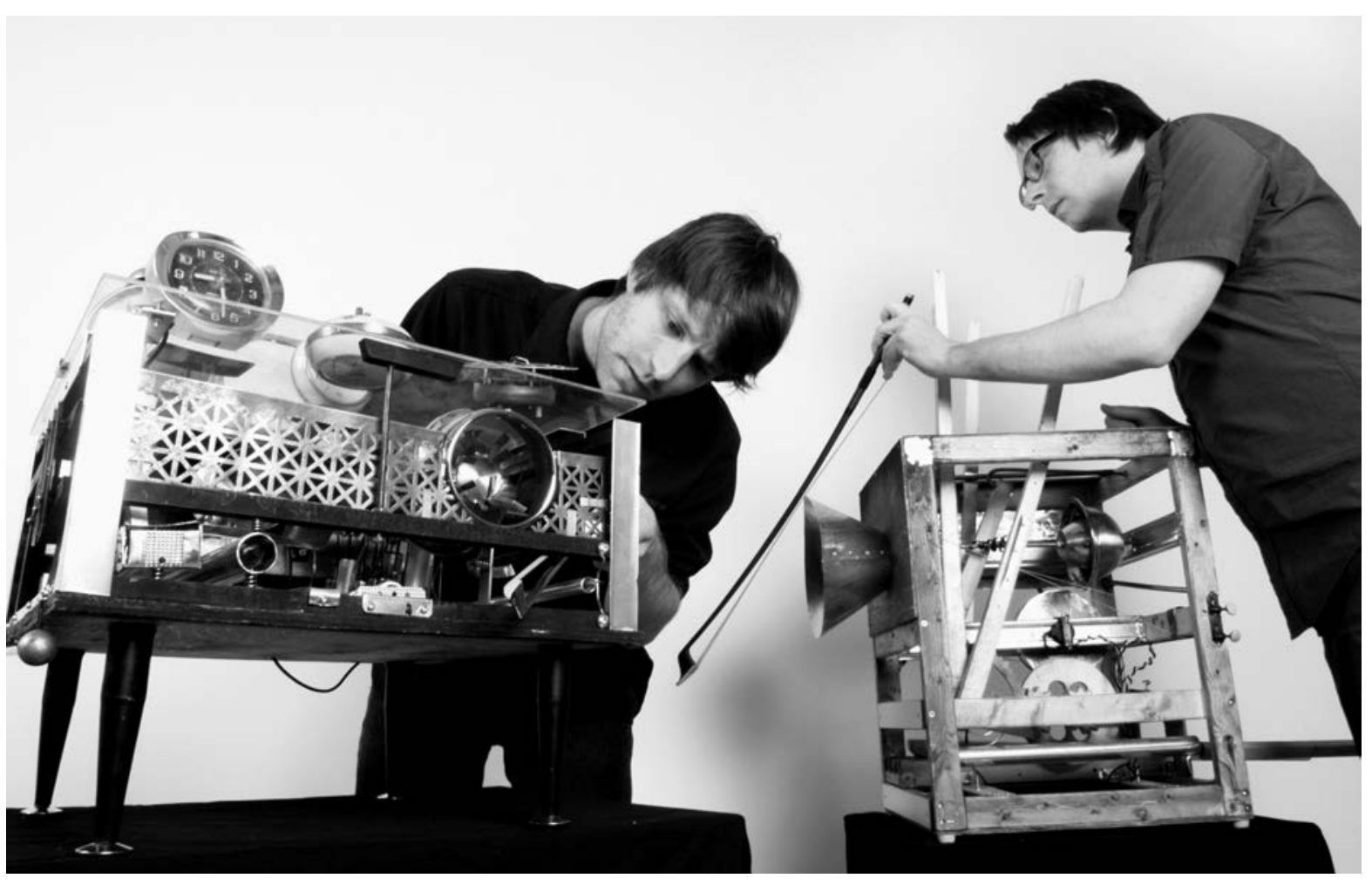

endroit de l'échelle temporelle, permettant de garder la précision voulue dans la composition. On y trouve donc une partie fixée ne laissant que peu de place pour l'improvisation.

S'il y a cette idée romantique dans la recherche électroacoustique d'un certain retour au tout-gestuel où chaque son trouverait son corréla physique, il serait malheureux de reléguer aux oubliettes la principale découverte apportée par la musique concrète: le son fixé. La tradition purement instrumentale subsistera toujours et doit faire partie de la recherche électroacoustique, mais comment nier la pertinence du travail de la matière sonore qui se fait en différé, légitimement dissocié du geste instrumental? Le musicien Adam Lindson explique :

Since music performance is a meaningful human activity, it makes little sense to consider music-making solely in terms of the physical production of sound. The context for the intention to make a sound in a musical performance is a human situation, while the mechanics of the sound production are part of a physical system ${ }^{34}$. 
William Brent est également de l'avis que nous devons aujourd'hui accepter que le son n'est pas toujours produit par son corréla mécanique:

One common assumption is particularly relevant: that there should be a perceivable correlation between the movements of a performing musician and the resulting sound. [...] Thus, we must ask : are desires for clear action-sound relationships merely historical remnants linked to a perverse obsession with technical prowess ${ }^{35}$ ?

Le fait de préserver une partie fixée sur support dans une performance permet aussi de laisser aux interprètes l'espace mental nécessaire au jeu, au geste et à la présence du corps.

En contrepoids à la partie fixe, une décision particulière a été prise lors de la conception de La chambre des machines : celle d'éradiquer l'ordinateur de la scène. Plutôt que de le mettre en scène comme un instrument, nous privilégions une approche où celui-ci est dissimulé en coulisse, évitant toute distraction informatique de la part des interprètes.

\section{Conclusion}

Des liens existent vraisemblablement entre l'objet sonore, l'objet matériel, le corps sonore, le corps humain, la démarche concrète et la démarche instrumentale. Nous sommes aujourd'hui à un point de l'histoire où les multiples stratégies de composition électroacoustiques développées au cours des soixante dernières années sont assez mûres pour le dialogue. L'utilisation de l'ordinateur pour la performance, le travail du son fixé, la manipulation d'objets, l'utilisation de la nouvelle lutherie sont tous autant d'ingrédients pertinents dans la création sonore contemporaine. Pierre Alexandre Tremblay note d'ailleurs que «cet alliage des différentes techniques est déjà communément utilisé dans les musiques populaires ». Il ajoute: «Pensons aux sections de percussions qui déclenchent des séquences sonores, aux doublons automatisés (guitares, cordes, etc.), le tout pouvant être synchronisé sur des séquences d'éclairage, etc. ${ }^{36}$ »

Après être passé par des périodes d'une certaine radicalité - que ce soit la musique concrète/acousmatique, la laptop performance ou le retour à une pratique électroacoustique purement basée sur le geste -, un hybride entre les diverses approches à la performance sonore semble être aujourd'hui une voie intéressante à explorer. Le développement d'une virtuosité associée à la nouvelle lutherie continuera à constituer une part importante de la recherche dans la composition et l'interprétation de la musique électroacoustique, mais cela ne devrait pas se faire au détriment de l'important héritage laissé par la musique concrète.
35. Brent, 2011, p. 430.

36. Entretien électronique avec Pierre Alexandre Tremblay, 2012. 


\section{REMERCIEMENT}

Je tiens à remercier Pierre Alexandre Tremblay sans lequel ce texte n'aurait jamais vu le jour.

\section{B I B L I OG RAPHIE}

AlLARD, Dominique (2012), «De l'objet à la “chose” animée: le désenchantement de l'objet / From Object to Animated “Thing” : The Disenchantment of the Object, esse arts + opinions, $\mathrm{n}^{\circ} 75,4^{-11}$

BAUDriLlaRD, Jean (1968), Le système des objets, Paris, Gallimard.

BERNIER, Nicolas (2012), Entretien électronique avec Pierre Alexandre Tremblay.

Brent, William (2011), "Aspects of Gesture in Digital Musical Instrument Design », in International Computer Music Conference 2011, University of Huddersfield (31 juillet-5 août), p. $429-43^{6 .}$

Chion, Michel (dir.) (1977), «Le concert pourquoi? comment?», Cahiers recherche/musique, $\mathrm{n}^{\circ} 5$, Paris, INA-GRM.

Chion, Michel (1990), L'audio-vision: son et image au cinéma, Paris, Nathan.

Chion, Michel (1993a), Le Promeneur écoutant, Paris, Plume.

Chion, Michel (1993b), «The State of Musique Concrète», Contemporary Music Review, vol. 8, $\mathrm{n}^{\mathrm{o}} 1$, p. $5^{1-55}$.

Chion, Michel (2009), La musique concrète, art des sons fixés, Lyon, Mômeludies éditions.

Collins, Nicolas (2008), "Why Live? Performance in the Age of Digital Reproduction », Leonardo Music Journal, vol. 18, p. 7-8.

FLusSER, Vilém (1999), The Shape of Things: A Philosophy of Design, Londres, Reaktion Books.

Foucault, Michel (1966), «Le corps utopique», conférence radiophonique prononcée sur France Culture, Paris, 21 décembre. Disponible en ligne: <http://culturevisuelle.org/ imagination/2010/06/05/foucault-«-le-corps-utopique-»> (consulté le 4 février 2013).

Le Feuvre, Lisa (dir.) (2010), Failure, Londres, Whitechapel Gallery; Cambridge, MIT Press.

Linson, Adam (2011), «Unnecessary Constraints: A Challenge to Some Assumptions of Digital Musical Instrument Design », in International Computer Music Conference 2011, University of Huddersfield ( 31 juillet-5 août), p. 421-424.

Ostertag, Bob (2002), «Human Bodies, Computer Music», Leonardo Music Journal, vol. 12, p. 11-14.

Rault, Lucie (2000), Musical Instruments: A Worldwide Survey of Traditional Music-making, Londres, Thames \& Hudson.

Russolo, Luigi ([1913]1967), The Art of Noise: Futurist Manifesto 1913, New York, Something Else Press.

SALTER, Chris (2010), Entangled: Technology and the Transformation of Performance, Cambridge, MIT Press.

Schaeffer, Pierre (1966), Traité des objets musicaux, Paris, Seuil.

Young, Rob (dir.) (2002), Undercurrents: The Hidden Wiring of Modern Music, Londres, Continuum.

\section{DISCOGRAPHIE}

BERNIER, Nicolas (2012), Travaux mécaniques. empreintes DIGITALes IMED 12114. 